\title{
Urinary Prothrombin Fragment $1+2$ as an Endogenous Marker of Venous Thromboembolism
}

\author{
Fredrik Wexels ${ }^{1,5 *}$, Walter Jeske ${ }^{2}$, Ola E. Dahl ${ }^{3,4}$ and Jawed Fareed ${ }^{5}$ \\ ${ }^{1}$ Department of Radiology, Diakonhjemmet Hospital, Oslo, Norway \\ ${ }^{2}$ Department of Thoracic and Cardiovascular Surgery, Loyola University Medical Center, Chicago, USA \\ ${ }^{3}$ Centre of Medical Science, Education and Innovation, Innlandet Hospital Trust, Brumunddal, Norway \\ ${ }^{4}$ Thrombosis Research Institute, London, UK \\ ${ }^{5}$ Department of Pathology, Loyola University Medical Center, Chicago, USA
}

*Corresponding author: Fredrik Wexels, Department of Radiology, Diakonhjemmet Hospital, Oslo, Norway; Email: fredrik.wexels@diakonsyk.no

Received: November 19, 2019; Accepted: November 26, 2019; Published: November 28, 2019

\begin{abstract}
Hypercoagulability may lead to Venous Thromboembolism (VTE) which is a common and potentially fatal disease. The symptoms of VTE are often non-specific and imaging is needed to confirm the diagnosis. Clinical probability models together with a D-dimer test are used to reduce the number of unnecessary radiological procedures. Numerous assays to detect thrombin generation have been developed where D-dimer measured in plasma is regarded as the pretest gold standard. The aim of this manuscript is to outline the present knowledge of using urinary prothrombin fragment $1+2$ $(\mathrm{F} 1+2)$ as a marker to determine coagulation activation in patients at risk of Deep Vein Thrombosis (DVT) or Pulmonary Embolism (PE). Papers were identified by searching PubMed for studies in which F1 +2 were measured in urine to determine coagulation activity in patients at risk of venous thromboembolism. Urinary F1 +2 levels can be used to identify patients at risk of VTE after hip and knee replacement surgery. Further, it reflects thrombin generation in patients with imaging verified DVT. However, in patients with imaging verified PE, the F1 +2 levels were not increased compared to those without PE. Compared to D-dimer and F1 +2 measured in plasma, urinary F1 +2 was inferior at discriminating VTE. Contrary to the mentioned results, in one study on patients undergoing total hip arthroplasty, urinary F1 + 2 did not reflect post-operative coagulation activation. Urine may be an attractive substrate to detect ongoing coagulation activation. However, tests specifically meant for urine analyses must be further developed.
\end{abstract}

Keywords: Venous thromboembolism, Prothrombin Fragment $1+2$, Urine

\section{Introduction}

The major pathological determinants for venous thrombosis formation were postulated by Rudolph Virchow in 1856 and are known as Virchow's triad. These factors include vessel wall damage, alterations of blood flow with stasis and abnormalities in platelet, coagulation and fibrinolytic pathways [1]. Venous thromboembolism (VTE), a common and potentially fatal disease which is mainly caused by hypercoagulability, can manifest as deep vein thrombosis (DVT) or pulmonary embolism (PE) [2,3]. Venous thrombi most often occur in the deep leg veins at sites of pathological blood flow or venous stasis, in areas of endothelial damage and in valve pockets [4]. If a clot originates in or propagates to the popliteal vein or more proximal veins, there is an increased risk of embolization to the pulmonary arteries with subsequent variable degrees of obstruction [3].

The precise incidence of VTE is unknown but it is estimated that it affects between 1 and 2 per 1000 of the population annually in the U.S. and that one third of these patients are diagnosed with PE [5]. In 2007, Cohen et al. estimated the number of non-fatal symptomatic VTE events and VTE related deaths in the European Union to be 684,019 DVT events, 434,723 PEs and 543,545 VTE related deaths in a total population of 454.4 million [6]. Venous thromboembolism is a rare condition in children younger than 15 years $[7,8]$. The incidence of DVT and PE increases with age. For those 65-69 years of age, the incidence per 1000 person years is 1.8 . This increases to 3.1 per 1000 person years for those aged 85-89 years [9]. Due to increased use of sensitive imaging techniques which can detect smaller and often insignificant pulmonary emboli, the hospital admissions for this disease have doubled over the last decades [10].

Clinical signs and symptoms of VTE may be obscure. Calf pain, swelling, heat and tenderness are clinical signs of DVT while PE patients may present with dyspnea, chest pain, hemoptysis, hypotension and tachycardia [3]. However, the symptoms are nonspecific and DVT can resemble, for example, cellulitis and PE may be indistinguishable from myocardial infarction [10]. Due to the nonspecific symptoms, imaging is needed to confirm the diagnosis of DVT or PE. Compression Ultrasonography (CUS) has high diagnostic accuracy for DVT [11]. Computer Tomography (CT) and Magnetic Resonance Imaging (MRI) are alternative or complementary DVT modalities with accuracy similar to that of CUS $[12,13]$. The reference modality for PE diagnosis is CT angiography [14]. Ventilationperfusion lung scanning combined with chest X-ray is an alternative in patients who cannot undergo CT angiography such as pregnant women [15]. In order to reduce the number of negative imaging 
investigations, models based on clinical signs and patient history have been developed to categorize the probability that a patient has VTE before a confirmatory test is performed. In those patients with an unlikely clinical probability and a negative $\mathrm{D}$-dimer test, thrombosis can be excluded without additional imaging [16-18]. Over the years numerous thrombin generation biomarker tests have been developed as VTE pretests including prothrombin fragment $1+2$ $(\mathrm{F} 1+2)$, thrombin-antithrombin complex (TAT) and D-dimer levels measured in plasma. Tests such as thrombin generation, procoagulant phospholipid-dependent clotting time and soluble P-selectin are currently used in research to identify prothrombotic risk [19]. The aim of this manuscript is to outline the present knowledge related to the use of urine as a substrate to determine coagulation activity in patients with clinical risk of DVT and PE.

\section{Methods}

PubMed was searched using the terms prothrombin fragment 1 +2 , urine prothrombin fragment $1+2$ and coagulation activation detection in urine. The resulting manuscripts that related to patients with risk of DVT or PE or both were selected for a manual review.

\section{Prothrombin fragment $1+2$}

Prothrombin fragment $1+2$ is a non-thrombotic polypeptide which is cleaved from prothrombin during its conversion to thrombin. $\mathrm{F} 1+2$ is released into the blood stream where it has half-life of approximately 90 minutes $[20,21]$. Due to the low molecular weight of F1 $+2(31 \mathrm{kDa})$ it is excreted in the urine where it can be detected by Enzyme-Linked Immuno-Sorbent Assay (ELISA) [22,23].

\section{Results of the Clinical Trials on Urinary F1 + 2 Measurement in Various Studies}

Prothrombin fragments have been detected in urine for many years and been shown to correlate with clinical symptoms of coagulation system activation $[23,24]$.

Prothrombin fragment $1+2$ in urine as an indicator of sustained coagulation activation after total hip arthroplasty [25]

Patients undergoing Total Hip Arthroplasty (THA) were followed post-surgery to document the occurrence of Vascular Thrombotic Complication (VTC) events and deaths. Pre- and postoperative levels of urine F1 + 2 were measured. Increased urine levels of F1 + 2 were observed immediately after the surgery and reached a peak level on postoperative day 3 before decreasing toward day 7 and normalizing at follow-up on day $35 \pm 5$. A Receiver Operator Characteristic (ROC) curve with Area under the Curve (AUC) of urinary F1 +2 levels performed on postoperative day 5 showed that F1 +2 levels in urine could accurately discriminate patients with and without increased risk of developing a VTC. Levels of F1 +2 in urine were significantly higher in patients who developed a VTC or death compared to the event-free patients.

Differences in urinary prothrombin fragment $1+2$ levels after total hip replacement in relation to venous thromboembolism and bleeding events [26]
This study assessed whether urinary F1 +2 measurements could be useful in identifying the risk of VTE or bleeding events in patients undergoing Total Hip Replacement (THR) surgery. Significantly higher levels of urinary F1 +2 were observed on post-operative day 3 in the VTE group compared to the event-free patients. At the same time the urine levels of $\mathrm{F} 1+2$ in the bleeding group were significantly lower than in the event-free group. Finally, the urinary F1 +2 levels were significantly higher on day 3 in the patients with VTE compared to those with bleeding events.

Urinary prothrombin fragment $1+2$ in relation to development of non-symptomatic and symptomatic venous thromboembolic events following total knee replacement [27]

Urinary F1 + 2 were measured on consecutive days in patients undergoing Total Knee Replacement (TKR) surgery. Bilateral venography was performed postoperatively (day 5-9) and about half of the patients (140 of 282 patients) were diagnosed with a VTE. Compared to the event free patients, those diagnosed with VTE had significantly higher levels of urinary F1 +2 .

Thrombin split products (prothrombin fragment $1+2$ ) in urine in patients with suspected deep vein thrombosis admitted for radiological verification [28]

This study evaluated urine F1 +2 levels in patients with suspected DVT referred for radiological verification. Patients with imagingverified DVT (CUS supplemented with unilateral venography when inconclusive) had significantly higher urinary F1 +2 levels compared to those without, both in patients with, and without, known comorbidities. Although not statistically significant, levels of urine F1 + 2 were higher in patients with DVT symptoms of more than one week compared to those with shorter symptom duration.

Prothrombin fragment $1+2$ in urine as a marker on coagulation activity in patients with suspected pulmonary embolism [29]

A study which measured prothromin fragment $1+2$ levels in urine from non-selected patients with suspected PE referred for imaging confirmation with contrast enhanced CT pulmonary angiography. Patients with imaging-verified PE had increased, however, not statistically significant, levels of urinary F1 +2 compared to the PE negative patients. Patients with high embolic burden, i.e. pulmonary artery obstruction index (PAOI) $\geq 25 \%$, had two-fold higher, however not significant, levels of urinary levels of F1 +2 compared to those with a lower burden.

D-dimer and prothrombin fragment $1+2$ in urine and plasma in patients with clinically suspected venous thromboembolism [30]

D-dimer and F1 + 2 levels measured in plasma and urine from patients with suspected VTE were significantly higher in those with imaging confirmed VTE compared to those without. In addition, there was a significant and positive correlation between D-dimer and F1 + 2 levels in plasma and between F1 +2 in plasma and urine. D-dimer had better predictive value for VTE than plasma F1 +2 followed by urinary $\mathrm{F} 1+2$ and there was no overlap in the ROC curves. There was a large variation of $\mathrm{F} 1+2$ levels between the plasma and urine samples with about 10 -fold higher levels in plasma. 
Thrombin generation in patients with suspected venous thromboembolism [31]

Patients with imaging confirmed VTE had markedly higher levels of D-dimer, plasma F1 + 2 and urine F1 + 2 compared to VTE negative patients. Similar findings were observed for the ex vivo measured Lagtime (LT) and Time to Peak (TTP) derived from a thrombin generation assay. There were similar associations between plasma and urine $\mathrm{F} 1+2$ and patient characteristics and the measured ex vivo biomarkers.

Prothrombin fragment F1 2 in plasma and urine during total hip arthroplasty [32]

A study evaluating peri-operative levels of plasma and urinary $\mathrm{F} 1+2$ in patients undergoing THA was performed. None of the included patients had VTE or serious bleeding events. Plasma and urine F1 +2 levels were significantly increased post-operatively with normalization of plasma levels on post-operative day 1 while urine levels remained significantly increased. There was a poor statistical correlation between $\mathrm{F} 1+2$ levels in plasma and urine.

\section{Discussion}

Studies using urine as the matrix to determine or monitor the extent of coagulation activation are rather limited compared to the number of studies performed on plasma biomarkers. A study in 2007 indicated that urine can be used to monitor the postoperative coagulation activity after THA surgery and to identify in which patients thromboprophylaxis can be discontinued after the first week [25]. The following year a publication on VTE and bleeding events after THR surgery stated that measurement of urinary F1 +2 could discriminate patients at risk of a VTE or major or clinically relevant, non-major bleeding [26]. In 2011 a study on TKR surgery patients found considerably higher urinary F1 +2 levels in these patients compared to the previous THR study and the authors indicated that this was due to a more intense coagulation activation after TKR than THR surgery, probably due to more bone and soft tissue trauma $[25,27]$. Further, they concluded that by measuring F1 +2 in urine it was possible to identify those patients in need of continued thromboprophylaxis due to persistent coagulation activation [27]. In a study on patients with clinically suspected DVT it was shown that measurement of urinary F1 +2 had the potential to reflect thrombin generation in DVT positive patients and that a DVT per se was responsible for this increase in patients without known comorbidities. However, underlying procoagulant conditions tend to mask the thrombin formation caused by a DVT. The urinary F1 +2 levels in the DVT positive patients showed a tendency to vary through the pathophysiological course of thrombus formation [28]. Pulmonary embolism, in contrast to a DVT, did not significantly increase the levels of F1 +2 in the urine. A possible explanation for this observation was the vast number of underlying procoagulant conditions in the PE population that might have contributed to increased urine F1 + 2 baselines level and thus masked the additive coagulation event. In addition, with a short half-life, F1 +2 was likely measurable at the time of initial clot formation but had cleared by the time the clot embolized. Although insignificant, a high embolic burden increased measured urine F1 +2 levels indicating that thrombus burden did impact detected prothrombin fragment levels [29].

Compared to the gold-standard of biomarker pre-tests which is currently D-dimer, plasma F1 +2 showed inferior ability to discriminate a VTE followed by F1 +2 in urine. The F1 2 concentrations in urine were substantially lower compared to plasma, which might be due to urine dilution of F1 +2 or chemical and bacterial differences that decreased the ELISA kit sensitivity on the urine samples [30]. Urinary $\mathrm{F} 1+2$ levels reflected procoagulant conditions in the same manner as $\mathrm{F} 1+2$ in plasma and had similar association with measured ex vivo biomarkers. However, urinary F1 + 2 levels did not exhibit identical analytic sensitivity [31].

Contrary to the previous studies, a study on THR surgery patients published in 2017 showed increased post-operative levels of F1 + 2 in plasma and urine, however, the correlation was poor and urinary F1 +2 levels did not reflect coagulation activation post-operatively [32]. Thrombin measurements in urine have been reported for the diagnosis of crescenting glomerulonephritis [33]. Increased thrombin levels as measured by using amidolytic methods were associated with fibrin deposits in the kidney and other associated pathologic manifestations. Other biomarkers of thrombin generation including fibrin monomers, TAT and protein $\mathrm{C}$ cleavage peptide have been measured in plasma and may be of interest for urinary measurements [34]. Additionally, fibrinopeptide $\mathrm{B}$ measured in urine has shown promising results to identify patients at risk of VTE [35].

\section{Conclusion}

The levels of $\mathrm{F} 1+2$ in plasma were about 10 -fold higher than corresponding urinary levels and plasma F1 +2 clearly had superior ability to determine whether or not a DVT or PE was present. Measurements of F1 +2 in urine was performed using ELISA kits designed for plasma analyses and the reason for its inferior performance may be that the sensitivity of the tests used is too low. However, we believe that with further development urine may be an attractive substrate to detect and determine ongoing coagulation.

\section{References}

1. Virchow R (1856) Gesammelte Abhandlungen zur Wissenschaftlichen Medizin. Meidinger; Frankfurt.

2. López JA, Kearon C, Lee AY (2004) Deep venous thrombosis. Hematology Am Soc Hematol Educ Program 2004: 439-456. [crossref]

3. Tapson VF (2008) Acute pulmonary embolism. N Engl J Med 358: 1037-1052. [crossref]

4. Nicolaides AN, Kakkar VV, Field ES, Renney JT (1971) The origin of deep vein thrombosis: a venographic study. Br J Radiol 44: 653-663. [crossref]

5. Beckman MG, Hooper WC, Critchley SE, Ortel TL (2010) Venous thromboembolism: a public health concern. American journal of preventive medicine 38: 495-501.

6. Cohen AT, Agnelli G, Anderson FA, Arcelus JI, Bergqvist D, et al. (2007) Venous thromboembolism (VTE) in Europe. The number of VTE events and associated morbidity and mortality. Thrombosis and haemostasis. 98: 756-764.

7. Heit JA, Silverstein MD, Mohr DN, Petterson TM, Lohse CM, et al. (2001) The epidemiology of venous thromboembolism in the community. Thromb Haemost 86: 452-463. [crossref]

8. Stein PD, Patel KC, Kalra NK, El Baage TY, Savarapu P, et al. (2002) Deep venous thrombosis in a general hospital. Chest 122: 960-962.

9. Kniffin WD Jr, Baron JA, Barrett J, Birkmeyer JD, Anderson FA Jr (1994) The epidemiology of diagnosed pulmonary embolism and deep venous thrombosis in the elderly. Archives of internal medicine 154: 861-866. 
10. Tritschler T, Kraaijpoel N, Le Gal G, Wells PS (2018) Venous Thromboembolism: Advances in Diagnosis and Treatment. Jama 320: 1583-1594.

11. Goodacre S, Sampson F, Thomas S, van Beek E, Sutton A (2005) Systematic review and meta-analysis of the diagnostic accuracy of ultrasonography for deep vein thrombosis. BMC medical imaging 5: 6.

12. Sampson FC, Goodacre SW, Thomas SM, van Beek EJ (2007) The accuracy of MRI in diagnosis of suspected deep vein thrombosis: systematic review and metaanalysis. European radiology 17: 175-181.

13. Thomas SM, Goodacre SW, Sampson FC, van Beek EJ (2008) Diagnostic value of CT for deep vein thrombosis: results of a systematic review and meta-analysis. Clinical radiology 63: 299-304.

14. van Beek EJ, Brouwerst EM, Song B, Stein PD, Oudkerk M (2001) Clinical validity of a normal pulmonary angiogram in patients with suspected pulmonary embolism-a critical review. Clin Radiol 56: 838-842. [crossref]

15. Sostman HD, Miniati M, Gottschalk A, Matta F, Stein PD, et al. (2008) Sensitivity and specificity of perfusion scintigraphy combined with chest radiography for acute pulmonary embolism in PIOPED II. Journal of nuclear medicine : official publication, Society of Nuclear Medicine 49: 1741-1748.

16. van der Hulle T, Dronkers CE, Klok FA, Huisman MV (2016) Recent developments in the diagnosis and treatment of pulmonary embolism. Journal of internal medicine 279: 16-29.

17. Wells PS, Anderson DR, Bormanis J, Guy F, Mitchell M, et al. (1997) Value of assessment of pretest probability of deep-vein thrombosis in clinical management. Lancet (London, England) 350: 1795-1798.

18. Wells PS, Anderson DR, Rodger M, Forgie M, Kearon C, et al. (2003) Evaluation of D-dimer in the diagnosis of suspected deep-vein thrombosis. N Engl J Med 349: 1227-1235. [crossref]

19. Riva N, Vella K, Hickey K, Bertu L, Zammit D, Spiteri S, et al. (2018) Biomarkers for the diagnosis of venous thromboembolism: D-dimer, thrombin generation, procoagulant phospholipid and soluble P-selectin. Journal of clinical pathology 71: 1015-1022.

20. Lippi G, Cervellin G, Franchini M, Favaloro EJ (2010) Biochemical markers for the diagnosis of venous thromboembolism: the past, present and future. Journal of thrombosis and thrombolysis 30: 459-471.

21. Páramo JA, Orbe J, Beloqui O, Benito A, Colina I, et al. (2004) Prothrombin fragment $1+2$ is associated with carotid intima-media thickness in subjects free of clinical cardiovascular disease. Stroke 35: 1085-1089. [crossref]

22. Lau HK, Rosenberg JS, Beeler DL, Rosenberg RD (1979) The isolation and characterization of a specific antibody population directed against the prothrombin activation fragments F2 and F1 + 2. J Biol Chem 254: 8751-8761. [crossref]

23. Lind SE, Goldshteyn S, Barry CP, Lindquist JR, Piergies AA, et al. (1999) Assessment of coagulation system activation using spot urine measurements. Blood coagulation \& fibrinolysis: an international journal in haemostasis and thrombosis 10: 285-289.

24. Bezeaud A, Aronson DL, Menache D, Guillin MC (1978) Identification of a prothrombin derivative in human urine. Thromb Res 13: 551-556. [crossref]

25. Borris LC, Breindahl M, Ryge C, Sommer HM, Lassen MR; $\mathrm{uF1+2}$ study group (2007) Prothrombin fragment 1+2 in urine as an indicator of sustained coagulation activation after total hip arthroplasty. Thromb Res 121: 369-376. [crossref]

26. Borris LC, Breindahl M, Lassen MR, Pap AF, Misselwitz F (2008) Differences in urinary prothrombin fragment $1+2$ levels after total hip replacement in relation to venous thromboembolism and bleeding events. Journal of thrombosis and haemostasis: JTH 6: 1671-1679.

27. Borris LC, Breindahl M, Lassen MR, Pap AF (2011) Urinary Prothrombin Fragment $1+2$ in relation to Development of Non-Symptomatic and Symptomatic Venous Thromboembolic Events following Total Knee Replacement. Thrombosis 201: 150750 .

28. Wexels F, Haslund A, Dahl OE, Pripp AH, Gudmundsen TE, et al (2013) Thrombin split products (prothrombin fragment $1+2$ ) in urine in patients with suspected deep vein thrombosis admitted for radiological verification. Thromb Res 131: 560-563. [crossref]

29. Wexels F, Dahl OE, Pripp AH, Seljeflot I, Borris LC, et al. (2014) Prothrombin fragment $1+2$ in urine as a marker on coagulation activity in patients with suspected pulmonary embolism. Thrombosis research 134: 68-71.

30. Wexels F, Seljeflot I, Pripp AH, Dahl OE (2016) D-Dimer and prothrombin fragment $1+2$ in urine and plasma in patients with clinically suspected venous thromboembolism. Blood coagulation \& fibrinolysis : an international journal in haemostasis and thrombosis 27: 396-400

31. Wexels F, Dahl OE, Pripp AH, Seljeflot I (2017) Thrombin Generation in Patients With Suspected Venous Thromboembolism. Clinical and applied thrombosis hemostasis: official journal of the International Academy of Clinical and Applied Thrombosis/Hemostasis 23: 416-421.

32. Borgen PO, Reikeras O (2017) Prothrombin fragment F1+2 in plasma and urine during total hip arthroplasty. Journal of orthopaedics 14: 475-479.

33. Kitamoto Y, Arizono K, Fukui H, Tomita K, Kitamura H, et al. (2015) Urinary thrombin: a novel marker of glomerular inflammation for the diagnosis of crescentic glomerulonephritis (prospective observational study). PloS one 10: 0118704.

34. Boisclair MD, Lane DA, Wilde JT, Ireland H, Preston FE, et al. (1990) A comparative evaluation of assays for markers of activated coagulation and/or fibrinolysis: thrombin-antithrombin complex, D-dimer and fibrinogen/fibrin fragment $\mathrm{E}$ antigen British journal of haematology 74: 471-479.

35. Morris TA, Marsh JJ, Burrows CM, Chiles PG, Konopka RG, et al. (2003) Urine and plasma levels of fibrinopeptide B in patients with deep vein thrombosis and pulmonary embolism. Thrombosis research 110: 159-165.

\section{Citation:}

Wexels F, Jeske W, Dahl OE, Fareed J (2019) Urinary Prothrombin Fragment $1+2$ as an Endogenous Marker of Venous Thromboembolism. Internal Med Res Open J Volume 4 (2): 1-4 